\title{
Analysis of reaction forces in human ankle joint during gait
}

\author{
Daniel Ganea, Elena Mereuta, Silvia Veresiu, Madalina Rus, and Valentin Amortila* \\ "Dunarea de Jos" University of Galati, Department of Mechanical Engineering, 111 Domnească \\ Street, Romania
}

\begin{abstract}
The paper aims to analyze the human ankle joint reaction forces with a multibody system approach, using MSC Adams software and its features. The paper presents the results provided by the simulation of a spatial mechanical structure, which replicates the human's locomotion system in terms of bone structure. The mechanical structure used for determining the human ankle joint reaction forces consists of 7 kinematic elements linked by 6 spherical joints constrained to 1 DOF each.
\end{abstract}

\section{Introduction}

The study of joints forces in human body joints is extremely important for physiotherapists, coaches and athletes. It is often difficult to determine these internal forces using noninvasive methods.

However, the inverse dynamics analysis can provide with good approximation the forces in human joints.

While direct dynamics is used for determining how human body actuators are used, the inverse dynamic analysis is widely used in estimating joints moments and reaction forces [1, 2].

Using the inverse dynamics approach in human body motion analysis the computational effort is considerable reduced by avoiding the numerical integration of state equations. Unfortunately, the approach is poorly explored in biomechanics [2,3].

The same method was use by some authors to estimate the force developed by human body actuators [4]. They have used a multibody model with 5 degrees of freedom.

Other authors [5] have analyzed the reaction force developed in the knee joint and also the force developed by human body actuators, using the inverse dynamic method combined with an MBS model.

The ankle is the anatomical structure of the human leg that connects two main kinematic elements that composes the human locomotor system. The first kinematic element is the shank formed by the tibia and the fibula. The second kinematic element is the foot, which is more complex, having 26 bones and 33 joints (Figure 1,2).

This kinematic chain is configured to ensure the stability and mobility of the human body on various surfaces during running, walking and even during orthostatic position [6].

\footnotetext{
* Corresponding author: valentin.amortila@ugal.ro
} 
Human gait is a semi-automatic action. This highly complex activity is unpredictable and automated. The aim of this article is to determine the reaction forces in the ankle joint, during a complete gait cycle, under three different loading cases. It is important to determine these forces not only for prosthesis design, but also for physiotherapists.

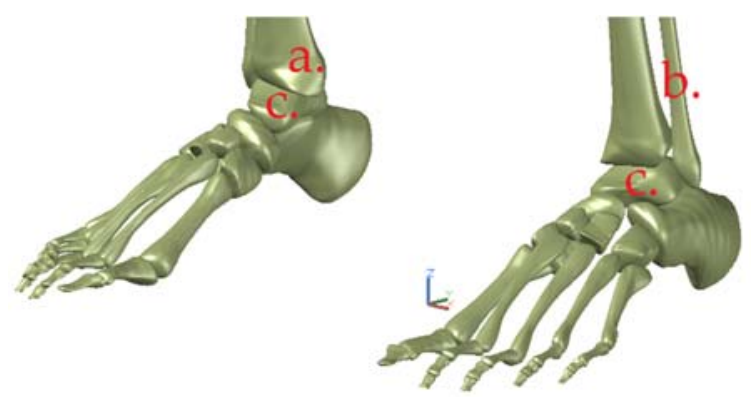

Fig. 1. Ankle joint: a. tibia bone; b. fibula bone; c. astragal bone.

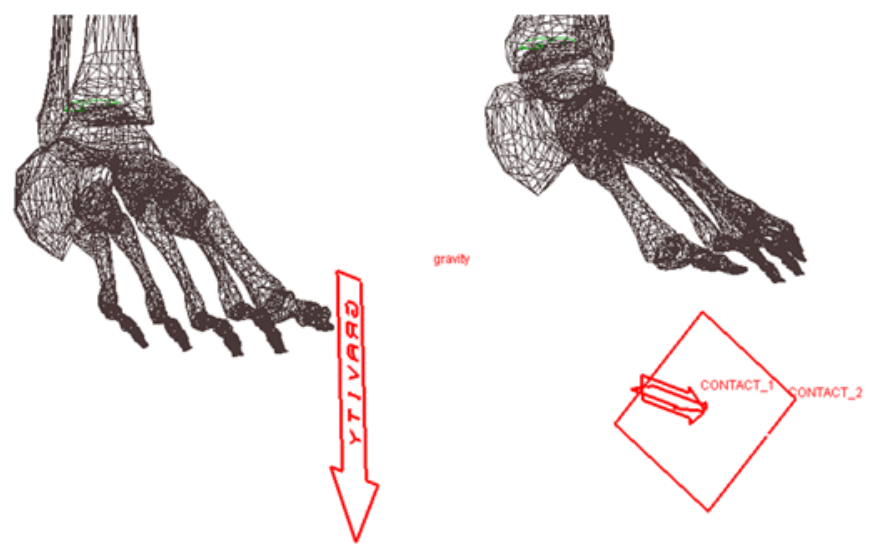

Fig. 2. Human ankle joint multibody system.

\section{Method}

The methods used in this study are mainly related to modelling and computer simulation. Due to the complexity of lower limb, some simplifying assumptions are required in order to facilitate the MBS modelling process. However, the simplifying assumptions have influence on the accuracy of the output data.

In order to get a more accurate model of the lower limb, we have imported a 3D geometric pattern available at 3Dlancer virtual library (http://3dlancer.net/en/3dmodel-humanskeleton-4792.html).

The imported 3D mesh model (Figure 3) was transformed into 3D solid using the Mesh to Solid feature of AutoCAD.

The solid thus created was imported in MSC Adams and the mechanical properties were associated to each kinematic element.

The kinematic structure is analyzed considering the following forces: the weight, the contact forces, the friction forces and applied forces that represent the supplementary load (Figure 4). 
As input data, we have considered also the time variation of six angular parameters, previously determined using a depth camera (Kinect).

A specially created computer application analyzes the images taken by the Kinect sensor and determines the coordinates of the main joints. It is also important to perform a kinematics analysis, which provides angular velocities and accelerations.

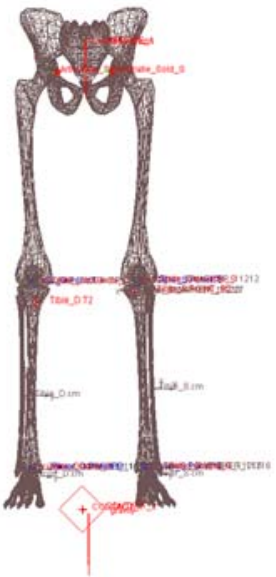

Fig. 3. 3D Mesh lower limb model.

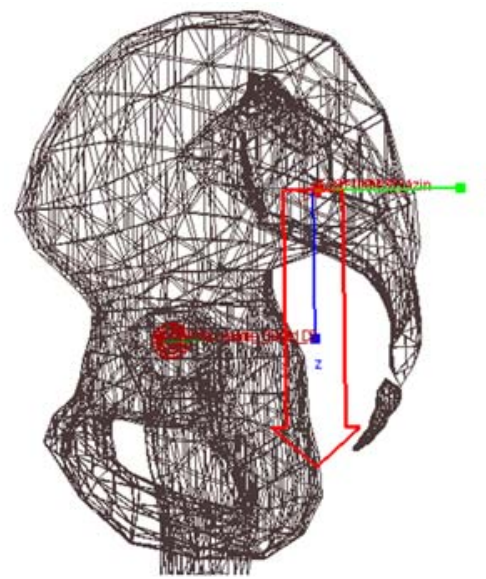

Fig. 4. Graphical representation of added weight.

The analysis was performed for a complete gait cycle, consisting of two steps, in 3 loading cases:

- $\quad$ First case - a normal gait cycle;

- $\quad$ Second case - a gait cycle with $34.46 \%$ of his body mass overload;

- Third case - a gait cycle with $64.93 \%$ of his body mass overload.

The gait cycle was divided into four stages (Figure 5). The first stage named start simulation includes two phases. The second stage is the support stage and includes 4 phases. The last two phases are the swing phase and stop simulation phase [6].

The dynamic analysis provides, besides the reaction forces the angular velocities and the angular accelerations for right ankle joint (RAJ) and left ankle joint (LAJ).

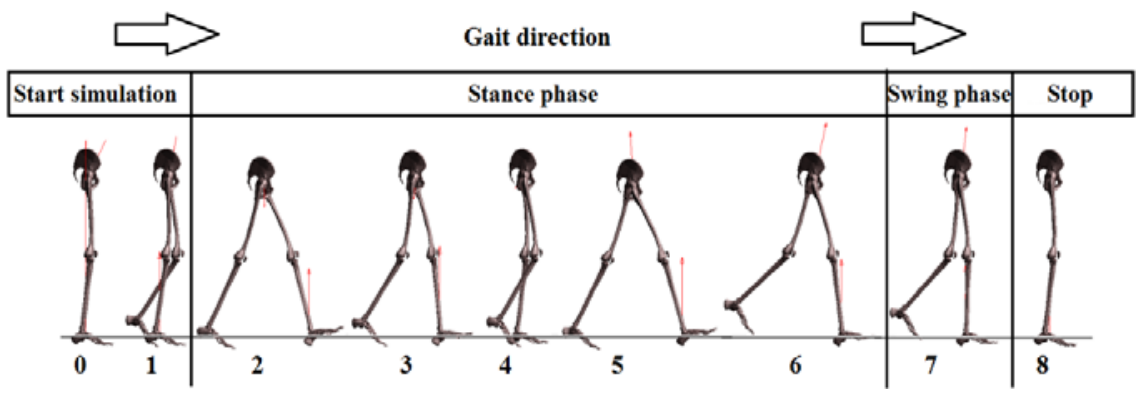

Fig. 5. Gait phases.

\section{Results}

The right ankle joint (RAJ) angular velocity has amplitude near $5 \mathrm{deg} / \mathrm{s}$. The right lower limb kinematic chain reaches this value during the sub - step of initial contact. For the second 
loading case, the right ankle joint (RAJ) angular velocity shows an increasing of amplitude, from $0.32 \mathrm{deg} / \mathrm{s}$ to $0.34 \mathrm{deg} / \mathrm{s}$ representing $6.25 \%$. For the third loading case the amplitude decreased by $20.5 \%$.

Relatively to right ankle joint (RAJ), the left ankle joint (LAJ) time variation of angular velocity reaches a smaller amplitude (of $1.87 \mathrm{deg} . / \mathrm{s}$ ), towards the end of sub - phase called support completion.

The left ankle joint (LAJ) average angular velocity during the first and second loading cases is $0.32 \mathrm{deg} / \mathrm{s}$. During the third loading case, the data reveals a decreasing of $15.62 \%$.

The right ankle joint (RAJ) time variation of angular acceleration amplitude is reached when the multibody passes from phase 1 to phase 2 (Figures 6, 7, 8).

For the first loading case, the angular acceleration amplitude is $6.46 \mathrm{deg} / \mathrm{s}^{2}$, for the second loading case the amplitude is $10.68 \mathrm{deg} / \mathrm{s}^{2}$, which increases with $95 \%$ during the third case $\left(20.83 \mathrm{deg} / \mathrm{s}^{2}\right)$. The average angular acceleration data shows an equal value in all three loading cases $\left(0.33\right.$ degrees $\left./ \mathrm{s}^{2}\right)$.

The LAJ angular acceleration variation with respect to time reaches its amplitude during the sub - phase called support completion (Figures 6, 7, 8).

During the first loading case the angular acceleration time variation curve with respect to time reaches a maximum value of $2.91 \mathrm{deg} / \mathrm{s}^{2}$, during the second case a rather small increasing to $3,01 \mathrm{deg} / \mathrm{s}^{2}(3.4 \%)$ and a significant increasing by $27.5 \%\left(3,84 \mathrm{degrees} / \mathrm{s}^{2}\right)$. The average angular acceleration shows a slight increasing from $0.12 \mathrm{deg} / \mathrm{s}^{2}$ to $0,138 \mathrm{deg} / \mathrm{s}^{2}$, during the second and third loading cases.

The simulation also provides information on the reaction forces, revealing that the ankle joints are stressed.

During the gait cycle, the reaction force time variation reaches its amplitude at the end of phase 2 of the first studied case (Figures 9, 10, 11).

During the second loading case, the reaction force reaches an amplitude increased by $90 \%$ $(1397.27 \mathrm{~N})$, while in the third loading case it increased by $12.26 \%(1568.6 \mathrm{~N})$.

The average reaction force recorded in the first loading case was $107.04 \mathrm{~N}, 180.03 \mathrm{~N}$ during the second one and $233.92 \mathrm{~N}$ during the third loading case.

The data reveals the fact that the most stressed joint is the LAJ. The amplitudes are the biggest during the beginning of the swing phase.

The LAJ reaction force is $852,154 \mathrm{~N}$ in the first loading case and the average is 204.05 $\mathrm{N}$. During the second loading case the reaction force has a maximum value of $1114 \mathrm{~N}$ and an average value of $315.09 \mathrm{~N}$.

The data resulted from the last loading case reveals that in this case the left ankle joint takes the biggest mechanical stress $(1631.6 \mathrm{~N}$ with an average of $410.39 \mathrm{~N})$.

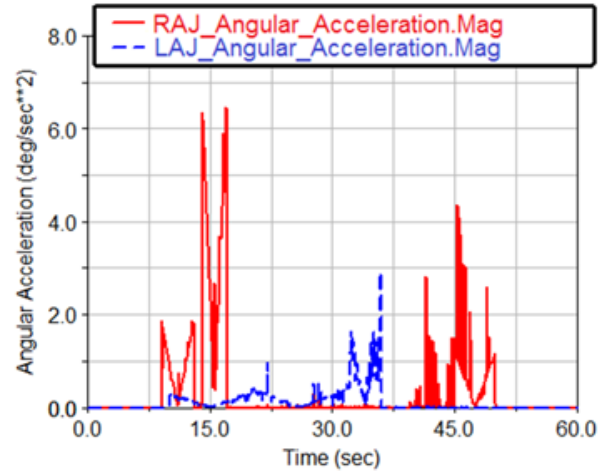

Fig. 6. RAJ and LAJ angular acceleration with respect to time (first loading case).

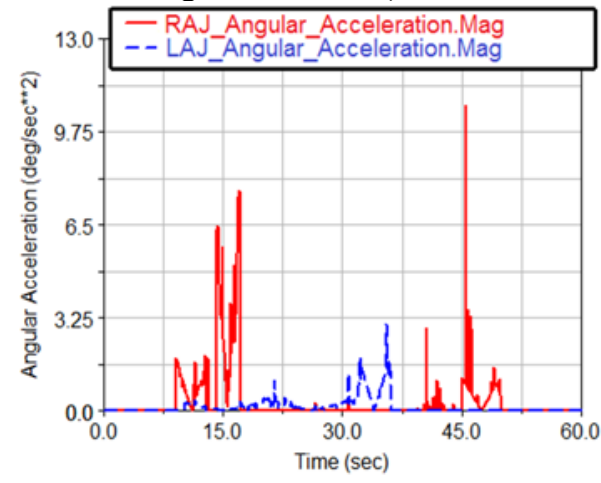

Fig. 7. RAJ and LAJ angular acceleration with respect to time (second loading case). 


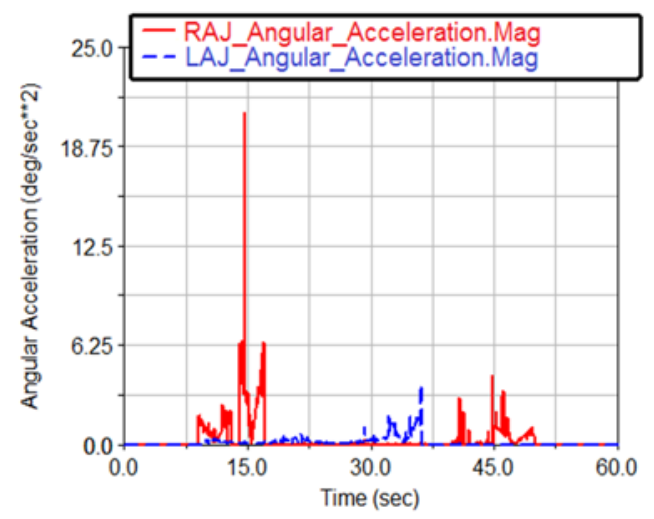

Fig. 8. RAJ and LAJ angular acceleration with respect to time (third loading case).

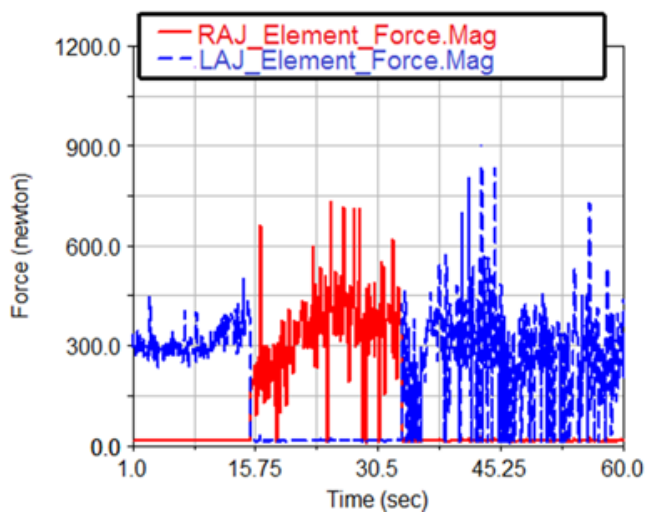

Fig. 9. Reaction force with respect to time recorded in RAJ and LAJ (first loading case).

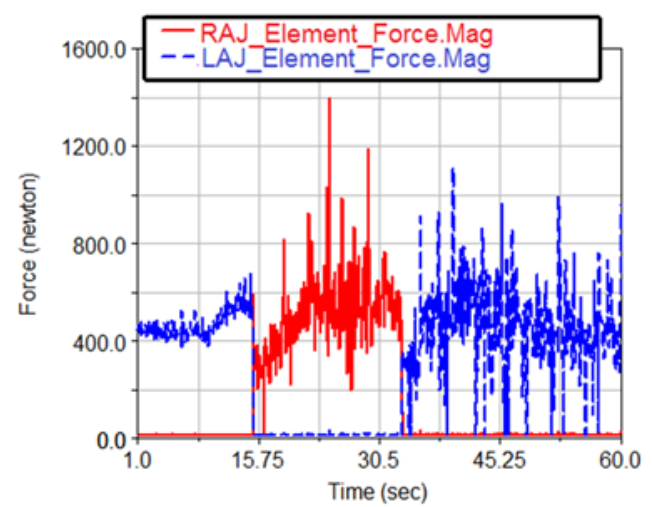

Fig. 10. Reaction force with respect to time recorded in RAJ and LAJ (second loading case). 


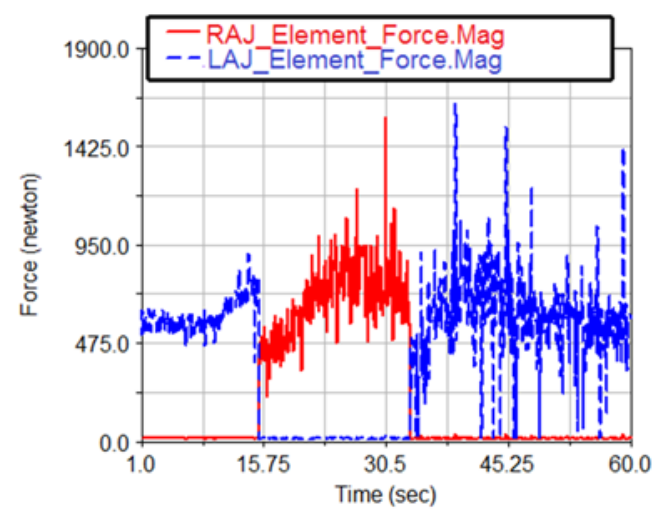

Fig. 11. Reaction force with respect to time recorded in RAJ and LAJ (third loading case).

\section{Conclusions}

The study of joints forces in human body joints is extremely important for physiotherapists, coaches and athletes. It is often difficult to determine these internal forces using noninvasive methods. However, the inverse dynamics analysis can provide with good approximation the forces in human joints.

Using the inverse dynamics approach in human body motion analysis the computational effort is considerable reduced by avoiding the numerical integration of state equations.

Simulating the model using laws of motion for the variation of kinematic parameters, we have been able to determine the reaction forces variation with respect to time for left and right ankle joints (LAJ, RAJ).

The simulated data revealed that the amplitude of the reaction force with respect to time for the right and left joint is asymmetrical.

For the first loading case, it can be observed that the reaction force has different amplitudes. Due to the applied forces, during the second loading case it is noticed that the mechanical stress rise with $90.04 \%$ for the RAJ and with $30.72 \%$ for the LAJ.

For the third loading case the data reveals that the reaction force increased significantly, to $1568.60 \mathrm{~N}(133.34 \%)$ for the RAJ and to $1631.60 \mathrm{~N}(91.46 \%)$ for LAJ.

To conclude, we were able to set a model which can be used in the study of forces that are developed during gait. This model can provide information on the internal forces which are important in prosthesis design. Also, the amplitudes of these forces are significant for optimal selection of more efficient endurance exercises for a better recovery of injured lower limbs.

\section{References}

1. S. Chowdhury, N. Kumar, Journal of Rehabilitation Robotics, 1, 2, (2013)

2. M. Ackermann, Dynamics and energetics of walking with prostheses, (2007)

3. D. Ganea, E. Mereuta, C. Mereuta, J AMM, 555 (2014)

4. F. Moissenet, L. Cheze, R. Dumas, J Multibody Syst Dyn, 28 (2012)

5. C.Y. Lin, P.J. Walter, S.A. Banks, G.M. Pandy, B.J. Fregly, J Biomech, 43 (2010)

6. C. L. Vaughan, B. L. Davis, J. C. O'Connor, Dynamics of Human Gait, (2 ${ }^{\text {nd }}$ Edition, Kiboho Publishers Cape Town, South Africa, 1999) 